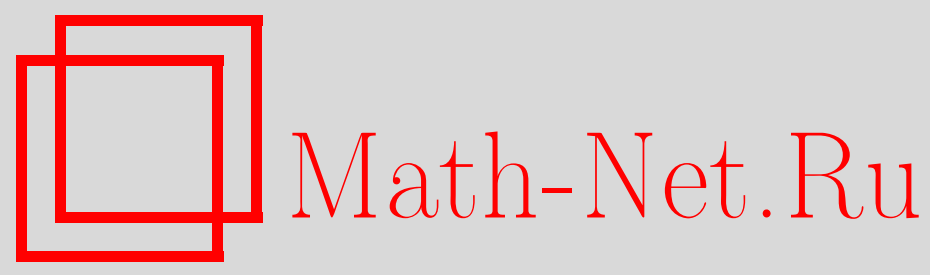

Т. О. Банах, Об одном кардинальном групповом инварианте, связанном с разбиениями абелевых групп, Матем. заметки, 1998, том 64, выпуск 3, 341-350

DOI: https://doi.org/10.4213/mzm1404

Использование Общероссийского математического портала Math-Net.Ru подразумевает, что вы прочитали и согласны с пользовательским соглашением http://www . mathnet.ru/rus/agreement

Параметры загрузки:

IP: 54.196 .121 .252

26 апреля 2023 г., 12:14:33

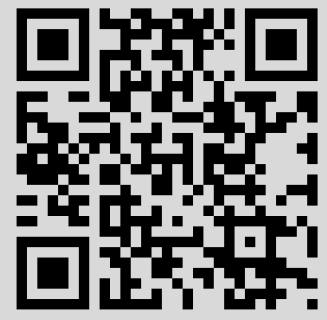




\title{
ОБ ОДНОМ КАР ДИНАЛЬНОМ ГРУППОВОМ ИНВАРИАНТЕ, СВЯЗАННОМ С РАЗБИЕНИЯМИ АБЕЛЕВЫХ ГРУПП
}

\author{
T. О. Банах
}

Для каждой абелевой группы $G$ вводится кардинальньй инвариант $\chi(G)$ и исследуются его свойства. В частном случае группы $G=\mathbb{Z}^{n}$ кардинал $\chi\left(\mathbb{Z}^{n}\right)$ равен минимальной мощности существенного подмножества в $\mathbb{Z}^{n}$, т.е. такого подмножества $A \subset \mathbb{Z}^{n}$, что для любой раскраски группы $\mathbb{Z}^{n}$ в $n$ цветов существует бесконечное одноцветное подмножество, симметричное относительно некоторой точки $\alpha$ из $A$. Доказьвается оценка $n(n+1) / 2 \leqslant \chi\left(\mathbb{Z}^{n}\right)<2^{n}$ для всех $n$, а также равенство $\chi\left(\mathbb{Z}^{n}\right)=n(n+1) / 2$ для $n \leqslant 3$. Полностью описана структура существенных подмножеств мощности $\chi\left(\mathbb{Z}^{n}\right)$ в $\mathbb{Z}^{n}$ для $n \leqslant 3$.

Библиограффия: 5 названий.

В работе [1] доказано, что для любого разбиения $\mathbb{Z}^{n}=A_{1} \cup \cdots \cup A_{n}$ группы $\mathbb{Z}^{n}$ на $n$ подмножеств существует такая точка $a \in \mathbb{Z}^{n}$ и бесконечное симметричное множество $S \subset \mathbb{Z}^{n}, S=-S$, что $a+S \subset A_{i}$ для некоторого $i$. И. В. Протасов отметил, что для случая $n=2$ точка $a$ может быть выбрана из любого наперед заданного подмножества $A \subset \mathbb{Z}^{n}$, содержащего $n+1$ аффинно независимые точки, и выдвинул гипотезу, что этот факт справедлив для любого $n$. В данной заметке мы покажем, что эта гипотеза Протасова неверна.

Мы посмотрим на проблему с несколько более общей точки зрения и для абелевых групп $G$ определим кардинальньй групповой инвариант $\chi(G)$. Все групшы, рассматриваемые в этой статье, предполагаются абелевыми. Напомним определение кардинала $\nu(G)$, введенного И.В. Протасовым в [2]. Для групшы $G \nu(G)$ есть минимальная мощность такого покрытия $\mathscr{U}$ группы, что пересечение $U \cap(2 g-U)$ конечно для любых $g \in G$ и $U \in \mathscr{U}$ (отметим, что условие конечности пересечения $U \cap(2 g-U)$ эквивалентно тому, что $g+S \not \subset U$ для любого бесконечного симметричного подмножества $S \subset G$, $S=-S)$. В [1] кардинал $\nu(G)$ вычислен для всех абелевых групп, причем

$\nu(G)=\left\{\begin{array}{l}r_{0}(G)+1, \text { если группа } G \text { конечно порождена, } \\ r_{0}(G)+2, \quad \text { если } G-\text { счетная бесконечно порожденная группа с }\left|[G]_{2}\right|<\aleph_{0}, \\ \max \left\{\left|[G]_{2}\right|, \log |G|\right\}, \quad \text { если } G \text { несчетна, либо }\left|[G]_{2}\right| \geqslant \aleph_{0},\end{array}\right.$

где $r_{0}(G)$ - свободньй ранг группы $G$ (см. [3]), $[G]_{2}=\{g \in G: 2 g=0\}$ - подгруппа элементов порядка 2 грушшы $G$ и $\log |G|=\min \left\{\gamma: 2^{\gamma} \geqslant|G|\right\}$. В частности, $\nu\left(\mathbb{Z}^{n}\right)=n+1$ для $n \in \mathbb{N}$.

Из определения кардинала $\nu(G)$ следует, что для любого покрытия $\mathscr{U}$ группы $G$ мощности $|\mathscr{U}|<\nu(G)$ существует такая точка $g \in G$ и множество $U \in \mathscr{U}$, что пересечение $U \cap(2 g-U)$ бесконечно. Назовем подмножество $A$ групшы $G$ существенны. 
любого покрытия $\mathscr{U}$ групшы $G$ мощности $|\mathcal{U}|<\nu(G)$ существует такая точка $a \in A$ и множество $U \in \mathscr{U}$, что пересечение $U \cap(2 a-U)$ бесконечно. Для группы $G$ определим кардинал $\chi(G)$ как минимальную мощность существенного подмножества групшы $G$ (определение корректно, поскольку множество $G$ всегда существенно в $G$ ). Отметим, что групша $G$ конечна тогда и лишь тогда, когда $\chi(G)=0$ (в этом случае существенным является пустое множество).

В связи с приведенными выше определениями возникают следующие общие проблемы:

А) описать структуру существенных подмножеств групшы $G$;

Б) вычислить кардинал $\chi(G)$ для конкретных груп;

В) для подгруппы $H \subset G$ установить соотношения между кардинальньпи числами $\chi(G), \chi(H)$ и $\chi(G / H)$.

Вопрос A) по-видимому сложен и на данный момент исчерпьвающий ответ на него известен только для групш $\mathbb{Z}$ и $\mathbb{Z}^{2}$ : подмножество группы $\mathbb{Z}$ (соответственно $\mathbb{Z}^{2}$ ) существенно тогда и только тогда, когда оно непусто (соответственно содержит три аффинно независимые точки).

Несколько лучше обстоит ситуация с вопросами Б) и В). Ниже через с обозначается мощность континуума.

ТЕОрема 1. Пусть $G$ - абелева группа мощности $|G| \leqslant \mathfrak{c . ~ Т о г д а ~}$

1) $\chi(G)=0$ в том и только том случае, когда группа $G$ конечна;

2) $\chi(G)=1$ в том и только том случае, когда либо $\nu(G)=2$, либо $\left|[G]_{2}\right| \geqslant \aleph_{0}$;

3) если группа $G$ конечно порохсдена, то $\chi(G)=\chi\left(\mathbb{Z}^{r_{0}(G)}\right)$;

4) если $G$ - бесконечно порохденная группа с $\nu(G)>2 u\left|[G]_{2}\right|<\aleph_{0}$, то $\chi(G)=\aleph_{0}$.

Таким образом, для конечно-порожденных абелевых групп $G$ вычисление кардинала $\chi(G)$ сводится к нахождению числа $\chi\left(\mathbb{Z}^{n}\right)$ для некоторого $n$. В этом плане мы располагаем следующей информацией.

Теорема 2. Для любого $n \in \mathbb{N}$

1) $\chi\left(\mathbb{Z}^{n}\right) \leqslant \chi\left(\mathbb{Z}^{n+1}\right)$;

2) $n(n+1) / 2 \leqslant \chi\left(\mathbb{Z}^{n}\right)<2^{n}$;

3) $\chi\left(\mathbb{Z}^{n}\right)=n(n+1) / 2$, если $n \leqslant 3$.

Отметим, что из теоремы 2 следует, что существенное подмножество групшы $\mathbb{Z}^{3}$ должно содержать как минимум шесть точек. Следовательно, гипотеза Протасова неверна.

Из формулы (1) и теорем 1, 2 непосредственно вытекает следующее утверждение, касающееся проблемы В).

Теорема 3. Если $G$ - группа мощности $|G| \leqslant \mathfrak{c}$ с конечным числом әлементов порядка 2, то для любой ее подгруппь $H \subset G$ имеют место неравенства $\chi(H) \leqslant \chi(G) u \chi(G / H) \leqslant \chi(G)$.

Отметим, что условие конечности подгрупш $[G]_{2}$ существенно, поскольку для группы $G=\mathbb{Z}_{2}^{\omega} \oplus \mathbb{Z}^{\omega}$ и ее подгрупп $H=\mathbb{Z}^{\omega}$ и $H^{\prime}=\mathbb{Z}_{2}^{\omega}$ имеем $\aleph_{0}=\chi(H)=\chi\left(G / H^{\prime}\right)$ $\not \chi(G)=1$.

Вернемся теперь к вопросу А) об описании структуры существенных подмножеств групп. Назовем подмножество $A$ группы $G$ минимальным существенным подмножеством, если $A$ не содержит собственных существенных подмножеств. Очевидно, что 
каждое конечное существенное множество содержит некоторое минимальное существенное подмножество, и что каждое конечное существенное подмножество мощности $\chi(G)$ минимально.

Треугольником в пространстве $\mathbb{R}^{n}$ мы назьваем любое множество, состоящее из трех аффинно независимых точек; октаәдром в $\mathbb{R}^{n}$ называется любое шестиэлементное подмножество вида $\left\{a \pm e_{1}, a \pm e_{2}, a \pm e_{3}\right\}$, где $a \in \mathbb{R}^{n}$ - некоторая точка и $e_{1}, e_{2}, e_{3}-$ три линейно независимых вектора в $\mathbb{R}^{n}$. Далее мы отождествляем $\mathbb{Z}^{n}$ с подмножеством $\mathbb{R}^{n}$.

ТеОремА 4. Справедливы следующие утверждения:

1) треугольники, и только они, являются минимальными существенными подмножествами группь $\mathbb{Z}^{2}$

2) каждое существенное подмножсество группы $\mathbb{Z}^{2}$ содержит треугольник;

3) октаэдры и только они являются минимальными существенными подмножествами мошности $\leqslant 6=\chi\left(\mathbb{Z}^{3}\right)$ группь $\mathbb{Z}^{3}$

4) существуют минимальные существенные конечные подмнохсества группь $\mathbb{Z}^{3}$, не являющиеся октаэдрами.

Для доказательства теоремы 1 нам понадобятся следующие две леммы.

ЛЕмма 1. Если $G$ - конечно порожденная группа, то $\chi(G)=\chi\left(\mathbb{Z}^{r_{0}(G)}\right)$.

ДоКАЗАТЕЛЬСтво. Согласно основной структурной теореме для конечно порожденных абелевых групп $[3,15.2]$ группа $G$ изоморфна прямой сумме $\mathbb{Z}^{r_{0}(G)} \oplus K$ для некоторой конечной групшы $K$. Далеемы отождествляем группу $G$ с $\mathbb{Z}^{r_{0}(G)} \oplus K$. Неравенство $\chi(G) \leqslant \chi\left(\mathbb{Z}^{r_{0}(G)}\right)$ очевидно, поскольку каждое существенное подмножество в $\mathbb{Z}^{r_{0}(G)}$ существенно в $\mathbb{Z}^{r_{0}(G)} \oplus K$ (здесь используется тот факт, что $\left.\nu(G)=\nu\left(\mathbb{Z}^{r_{0}(G)}\right)\right)$. Докажем обратное неравенство. Пусть $A$ - существенноеподмножество мощности $|A|=\chi(G)$ в $G=\mathbb{Z}^{r_{0}(G)} \oplus K$. Мы утверждаем, что проекция $\operatorname{pr}(A)$ множества $A$ на $\mathbb{Z}^{r_{0}(G)}$ существенна в $\mathbb{Z}^{r_{0}(G)}$. Пусть $\mathscr{U}$ - любое покрытие группы $\mathbb{Z}^{r_{0}(G)}$ мощности $|\mathscr{U}|\left\langle\nu\left(\mathbb{Z}^{r_{0}(G)}\right)=\right.$ $r_{0}(G)+1$. Тогда $\mathscr{U} \times K=\{U \times K \mid U \in \mathscr{U}\}$ - покрытие группы $G$ мощности $|\mathcal{U} \times K|<\nu(G)$. Поскольку $A \subset G$ - существенное подмножество, существует такая точка $a \in A$ и множество $U \in \mathscr{U}$, что пересечение $U \times K \cap(2 a-U \times K)$ бесконечно. Поскольку группа $K$ конечна и $U \times K \cap(2 a-U \times K) \subset(U \cap(2 \operatorname{pr}(a)-U)) \times K$, мы заключаем, что пересечение $U \cap(2 \operatorname{pr}(a)-U)$ тоже бесконечно. Иными словами, $\operatorname{pr}(A)-$ существенное подмножество $\mathbb{Z}^{r_{0}(G)}$ и, следовательно, $\chi\left(\mathbb{Z}^{r_{0}(G)}\right) \leqslant \chi(G)$.

Лемма 2. Пусть $G$ - бесконечно порожденная абелева группа мощности $|G| \leqslant \mathfrak{c}$ с $\nu(G)>2 u\left|[G]_{2}\right|<\aleph_{0} u H-$ конечно порохденная подгруппа в $G$. Тогда подмнохество $H$ несущественно в $G$.

ДокАЗАТЕЛЬСТво. Из формулы (1) следует, что $\nu(H)<\nu(G)$. Тогда из определения кардинала $\nu(H)$ и неравенств $\nu(H)<\nu(G)$ и $\nu(G)>2$ следует существование такого покрытия $\mathscr{U}=\left\{U_{1}, \ldots, U_{n}\right\}$ группы $H$, что $2 \leqslant|\mathcal{U}|<\nu(G)$ и для любых $h \in H$ и $U \in \mathscr{U}$ пересечение $U \cap(2 h-U)$ конечно. Пусть $[G / H]_{2}$ - множество элементов порядка 2 факторгруппы $G / H$. Тогда множество всех остальных элементов групшы $G / H$ можно представить в виде $(G / H) \backslash[G / H]_{2}=-R \cup R$, где $R \subset G / H$ - такое множество, что $-R \cup R=\varnothing$. Пусть $s-$ любое сечение проекции $\pi: G \rightarrow G / H$, т.е. такое отображение $s: G / H \rightarrow G$, что $\pi \circ s=\mathrm{id}$. Определим покрытие $\mathscr{V}=\left\{V_{1}, \ldots, V_{n}\right\}$ грушшы $G$, полагая

$$
\begin{gathered}
V_{1}=(s(R)+H) \cup\left(s\left([G / H]_{2}\right)+U_{1}\right), \quad V_{2}=(s(-R)+H) \cup\left(s\left([G / H]_{2}\right)+U_{2}\right), \\
V_{i}=s\left([G / H]_{2}\right)+U_{i}, \quad 2<i \leqslant n .
\end{gathered}
$$


Несложно проверить, что для любого $h \in H$ и любого $i \in\{1, \ldots, n\}$ пересечение $V_{i} \cap\left(2 h-V_{i}\right)$ конечно. Поскольку $|\mathcal{V}|=|\mathcal{U}|<\nu(G)$, множество $H$ несущественно в $G$.

ДокАЗАТЕЛЬСтво тЕоремы 1. Утверждение 3) очевидным образом следует из леммы 1. Докажем утверждение 4). Пусть $G$-бесконечно порожденная групша с $\nu(G)>2$ и $\left|[G]_{2}\right|<\aleph_{0}$. По формуле $(1) \nu(G) \leqslant \aleph_{0}$ и по лемме $2 \chi(G) \geqslant \aleph_{0}$. Для доказательства обратного неравенства зафиксируем счетную бесконечно-порожденную подгруппу $H \subset G$ c $r_{0}(H)=\min \left\{\aleph_{0}, r_{0}(G)\right\}$. Покажем, что множество $H$ существенно в $G$. Пусть $\mathscr{U}-$ покрытие группы $G$ мощности $|\mathscr{U}|<\nu(G)$. Тогда $\mathscr{U} \cap H=\{U \cap H \mid U \in \mathscr{U}\}$ - покрытие группы $H$ мощности $|\mathcal{U} \cap H| \leqslant|\mathcal{U}|$. По формуле $(1) \nu(G)=\min \left\{\aleph_{0}, r_{0}(G)+2\right\}=$ $\min \left\{\aleph_{0}, r_{0}(H)+2\right\}=\nu(H)$. Поскольку $|\mathcal{U} \cap H|<\nu(G)=\nu(H)$, по определению кардинала $\nu(H)$ существует точка $h \in H$ и множество $U \in \mathscr{U}$ такие, что пересечение $(H \cap U) \cap(2 h-H \cap U)$ бесконечно. Но тогда пересечение $U \cap(2 h-U)$ тоже бесконечно. Таким образом, счетное множество $H$ существенно в $G$. Следовательно, $\chi(G) \leqslant \aleph_{0}$.

Утверждение 1) следует из определения кардиналов $\nu(G)$ и $\chi(G)$. Наконец, утверждение 2) легко выводится из утверждений 1$), 3), 4)$ и очевидного неравенства $\chi\left(\mathbb{Z}^{n}\right)>1$ для $n>1$.

Доказательство теоремы 2 разобьем на ряд лемм.

Для вектора $\ell$ евклидова пространства $\mathbb{R}^{n}$ через $\ell^{\perp}$ обозначается гиперплоскость в $\mathbb{R}^{n}$, проходящая через 0 и перпендикулярная вектору $\ell$.

Лемма 3. Для любого подмножсества $A \subset \mathbb{R}^{n}$ мощности $|A|<n(n+1) / 2 u$ любого конечного множсества $B \subset \mathbb{R}^{n}$ существует разбиение $\mathbb{R}^{n}=A_{1} \cup \cdots \cup A_{n} u$ вектор $\ell \in \mathbb{R}^{n}$ такие, что

i) для любых $a \in A$ u $i \in\{1, \ldots, n\}$ пересечение $A_{i} \cap\left(2 a-A_{i}\right)$ ограничено;

ii) для любих $b \in B u i \in\{1, \ldots, n\}$ проекиия множества $A_{i} \cap\left(2 b-A_{i}\right)$ на гиперплоскость $\ell^{\perp}$ ограничена.

ДокАЗАТЕЛЬСтво. Для $n=1$ лемма очевидна. Предположим, что лемма 3 верна для всех $k \leqslant n$. Покажем, что она верна для $k=n+1$. Зафиксируем множество $A \subset \mathbb{R}^{n+1}$ мощности $|A|<(n+1)(n+2) / 2$ и конечное подмножество $B \subset \mathbb{R}^{n+1}$. Без ограничения общности можно считать, что $|A|=(n+1)(n+2) / 2-1$. Обозначим через $\operatorname{conv}(A)$ вьпуклую оболочку множества $A$ в $\mathbb{R}^{n+1}$. Для выпуклого многогранника $\operatorname{conv}(A)$ нетрудно найти опорную гиперплоскость $s$, содержащую не менее $n+2$ точек множества $A$. Тогда за пределами гиперплоскости $s$ останется менее $(n+1)(n+2) / 2-$ $(n+2)=n(n+1) / 2$ точек множества $A$. Сделав соответствующее ортогональное аффинноепреобразование пространства $\mathbb{R}^{n+1}$, мы можем считать, что гиперплоскость $s$ совпадает с подпространством $\mathbb{R}^{n}=\left\{\left(x_{1}, \ldots, x_{n+1}\right) \in \mathbb{R}^{n+1} \mid x_{n+1}=0\right\}$, а множество $A \backslash s$ содержится в полупространстве $\left\{\left(x_{1}, \ldots, x_{n+1}\right) \in \mathbb{R}^{n+1} \mid x_{n+1}<0\right\}$. Обозначим через $\operatorname{pr}_{n}: \mathbb{R}^{n+1} \rightarrow \mathbb{R}^{n}=s$ проекцию на первые $n$ координат и положим $A^{\prime}=\operatorname{pr}_{n}(A \backslash s)$ и $B^{\prime}=\operatorname{pr}_{n}(A \cup B)$. Согласно индуктивному предположению существует разбиение $\mathbb{R}^{n}=A_{1}^{\prime} \cup \cdots \cup A_{n}^{\prime}$ и вектор $\ell \in \mathbb{R}^{n}$ такие, что

$\left.\mathrm{i}^{\prime}\right)$ для любых $a \in A^{\prime}$ и $1 \leqslant i \leqslant n$ пересечение $A_{i}^{\prime} \cap\left(2 a-A_{i}^{\prime}\right)$ ограничено;

$\left.\mathrm{ii}^{\prime}\right)$ для любых $b \in B^{\prime}$ и $1 \leqslant i \leqslant n$ проекция множества $A_{i}^{\prime} \cap\left(2 b-A_{i}^{\prime}\right)$ на гиперплоскость $l^{\perp} \subset \mathbb{R}^{n}$ ограничена.

Без ограничения общности $\ell=(0, \ldots, 0,1,0)$ и $\ell^{\perp}=\mathbb{R}^{n-1}=\left\{\left(x_{1}, \ldots, x_{n+1}\right) \in \mathbb{R}^{n+1}\right.$ | $\left.x_{n}=x_{n+1}=0\right\}$. Обозначим через $\mathrm{pr}_{n-1}: \mathbb{R}^{n+1} \rightarrow \mathbb{R}^{n-1}=l^{\perp}$ (ортогональную) 
проекцию на первые $n-1$ координат. Из условия iі') следует существование такого числа $R>0$, что

$$
\bigcup_{\substack{b \in B^{\prime} \\ 1 \leqslant i \leqslant n}} \operatorname{pr}_{n-1}\left(A_{i}^{\prime} \cap\left(2 b-A_{i}^{\prime}\right)\right) \subset[-R, R]^{n-1} \subset \mathbb{R}^{n-1} .
$$

Обозначим через $\mathrm{pr}_{\ell}: \mathbb{R}^{n+1} \rightarrow \mathbb{R}=\mathbb{R} \cdot \ell$ проекцию на $n$-ю координату и положим $m=\min _{b \in B^{\prime}} \operatorname{pr}_{\ell}(b)$. Искомое разбиение $\mathbb{R}^{n+1}=A_{0} \cup \cdots \cup A_{n}$ определим следующим образом:

$$
\begin{gathered}
A_{0}=[-R, R]^{n-1} \times(-\infty, m) \times\{0\} \cup \bigcup_{t>0}[-R-t, R+t]^{n-1} \times \mathbb{R} \times\{t\}, \\
A_{i}=\left(A_{i}^{\prime} \times \mathbb{R}\right) \backslash A_{0}, \quad 1 \leqslant i \leqslant n .
\end{gathered}
$$

Можно проверить, что это разбиение и вектор $\ell$ удовлетворяют условиям i), ii) леммы 3.

Лемма 4. Для любого $n \in \mathbb{N}$ имеет место неравенство $n(n+1) / 2 \leqslant \chi\left(\mathbb{Z}^{n}\right)<2^{n}$.

ДокАЗАТЕЛЬСтво. Неравенство $n(n+1) / 2 \leqslant \chi\left(\mathbb{Z}^{n}\right)$ очевидным образом следует из леммы 3. Для доказательства неравенства $\chi\left(\mathbb{Z}^{n}\right)<2^{n}$ покажем, что множество $E=\{0,1\}^{n} \backslash\{1\}^{n}$ существенно в $\mathbb{Z}^{n}$. Предположим, что это не так. Тогда существует разбиение $\mathbb{Z}^{n}=A_{1} \cup \cdots \cup A_{n}$ такое, что для любых $a \in E$ и $1 \leqslant i \leqslant n$ пересечение $A_{i} \cap\left(2 a-A_{i}\right)$ конечно. Пусть $R$ - положительное четное число такое, что

$$
\bigcup_{\substack{a \in E \\ 1 \leqslant i \leqslant n}} A_{i} \cap\left(2 a-A_{i}\right) \subset(-R, R)^{n} .
$$

Обозначим через $K n$-мерньй куб $[-R, R]^{n} \subset \mathbb{R}^{n}$ и через $\partial K$ его границу. Разобьем куб $K$ на $n$-мерные кубики вида $x+[0,2]^{n}$, где $x \in(2 \mathbb{Z})^{n}$. Далее, каждьй такой кубик триангулируем так, чтобы вершины каждого симплекса этой триангуляции принадлежали множеству $(2 \mathbb{Z})^{n}$ и были линейно упорядочены относительно естественного частичного порядка “ $\leqslant$ " на $(2 \mathbb{Z})^{n}$ (отметим, что такая триангуляция единственна). Таким образом, мы определяем некую триангулящию $T$ куба $K$.

Обозначим через $\Delta(n-1)$-мерньй симплекс с вершинами $v_{1}, \ldots, v_{n}$. Построим кусочно-линейное отображение $f: K \rightarrow \Delta$, определив его в вершинах триангулящии формулой $f(v)=v_{i}$, где $i$ выбирается из условия $v \in A_{i}$.

Зафиксируем любую точку $x \in \partial K$ и покажем, что $f(-x) \neq f(x)$. Пусть $\sigma-$ минимальньй симплекс триангуляции $T$, содержащий точку $x$, и пусть $x_{1} \leqslant \cdots \leqslant x_{k}-$ его вершины, линейно упорядоченные относительно частичного порядка "\$” на $\mathbb{Z}^{n}$. Пусть $i \in\{1, \ldots, n\}$ - цвет вершины $x_{k}$, т.е. такое $i$, что $x_{k} \in A_{i}$. Из минимальности $\sigma$ следует, что $x$ - внутренняя точка симплекса $\sigma$, а из определения отображения $f$, что $f(x) \in \Delta \backslash \Delta_{i}$, где $\Delta_{i}-(n-2)$-мерная грань симплекса $\Delta$, не содержащая вершины $v_{i}$.

Пусть $\tau$ - минимальный симплекс триангуляции $T$, содержащий точку $-x$. Отметим, что $\sigma, \tau \subset \partial K$. Нетрудно проверить, что $\tau^{(0)} \subset 2 E-x_{k}$, где $\tau^{(0)}$ обозначает множество вершин симплекса $\tau$. Поскольку $x_{k} \in A_{i} \cap \partial K$, из соотношения (2) вытекает, что $\tau^{(0)} \subset \mathbb{Z}^{n} \backslash A_{i}$. Тогда по определению отображения $f f(-x) \in \Delta_{i}$. Следовательно, $f(-x) \neq f(x)$.

Поскольку $\partial K$ является топологической $(n-1)$-мерной сферой и $\Delta$ вкладьвается в $\mathbb{R}^{n-1}$, из теоремы Борсука-Улама об антиподах $[4$, гл. V,$\S 8.9]$ следует существование такого $x \in \partial K$, что $f(x)=f(-x)$. Полученное противоречие завершает доказательстBO. 
Лемма 5. Для любого $n \in \mathbb{N}$ имеет место неравенство $\chi\left(\mathbb{Z}^{n}\right) \leqslant \chi\left(\mathbb{Z}^{n+1}\right)$.

ДокАЗАтЕльство. Запишем $\mathbb{Z}^{n}$ в виде прямой суммы $\mathbb{Z}^{n} \oplus \mathbb{Z}$ и обозначим через $\operatorname{pr}_{1}: \mathbb{Z}^{n+1} \rightarrow \mathbb{Z}^{n}$ и $\mathrm{pr}_{2}: \mathbb{Z}^{n+1} \rightarrow \mathbb{Z}$ естественные проекции. Пусть $A$ - существенное подмножество в $\mathbb{Z}^{n+1}$ мощности $|A|=\chi\left(\mathbb{Z}^{n+1}\right)$. В силу леммы 4 кардинал $|A|$ конечен. Покажем, что $\operatorname{pr}_{1}(A)$ - существенное множество в $\mathbb{Z}^{n}$. Для этого зафиксируем произвольное покрытие $\mathscr{U}=\left\{U_{1}, \ldots, U_{n}\right\}$ групшы $\mathbb{Z}^{n}$ мощности $|\mathscr{U}|<\nu\left(\mathbb{Z}^{n}\right)=n+1$. Пусть $m=\max _{a \in A} \operatorname{pr}_{2}(a)$. Положим $V_{0}=\mathbb{Z}^{n} \times(\mathbb{Z} \cap(m, \infty)) \subset \mathbb{Z}^{n+1}$ и $V_{i}=\left(U_{i} \times \mathbb{Z}\right) \backslash V_{0}$, $1 \leqslant i \leqslant n$. Очевидно, что $\mathscr{V}$ - покрытие группы $\mathbb{Z}^{n+1}$ мощности $|\mathscr{V}|=n+1<\nu\left(\mathbb{Z}^{n+1}\right)$. Поскольку подмножество $A \subset \mathbb{Z}^{n+1}$ сушественно, пересечение $V_{i} \cap\left(2 a-V_{i}\right)$ бесконечно для некоторой точки $a \in A$ и индекса $i \in\{0, \ldots, n\}$. По определению множества $V_{0}$ пересечение $V_{0} \cap\left(2 a-V_{0}\right)$ пусто для любого $a \in A$. Следовательно, $i \neq 0$. Пусть $(x, y) \in \mathbb{Z}^{n} \oplus \mathbb{Z}$ - координаты точки $a$. Из определения множества $V_{i}$ следует, что $V_{i} \cap\left(2 a-V_{i}\right) \subset\left(U_{i} \cap\left(2 x-U_{i}\right)\right) \times(\mathbb{Z} \cap[2 y-m, m])$. Поскольку "отрезок" $\mathbb{Z} \cap[2 y-m, m]$ содержит конечное число точек, пересечение $U_{i} \cap\left(2 x-U_{i}\right)$ должно быть бесконечным. Стало быть, множество $\operatorname{pr}(A) \ni x$ существенно в $\mathbb{Z}^{n}$ и $\chi\left(\mathbb{Z}^{n}\right) \leqslant|\operatorname{pr}(A)| \leqslant|A|=\chi\left(\mathbb{Z}^{n+1}\right)$.

Лемма 6. Имеет место равенство $\chi\left(\mathbb{Z}^{3}\right)=6$.

ДокАЗАтЕльство. Неравенство $\chi\left(\mathbb{Z}^{3}\right) \geqslant 6$ следует из леммы 4 . Для доказательства обратного неравенства, покажем, что шестиэлементное подмножество $E=\{0,1\}^{3} \backslash$ $\{(0,1,1),(1,0,0)\}$ существенно в $\mathbb{Z}^{3}$. Предположим, что это не так. Тогда существует разбиение $\mathbb{Z}^{3}=A_{1} \cup A_{2} \cup A_{3}$ такое, что для любых $a \in E$ и $i \in\{1,2,3\}$ пересечение $A_{i} \cap\left(2 a-A_{i}\right)$ конечно. Пусть $R$ - положительное четное число такое, что

$$
\bigcup_{\substack{a \in E \\ 1 \leqslant i \leqslant 3}} A_{i} \cap\left(2 a-A_{i}\right) \subset(-R, R)^{3} .
$$

Обозначим через $K$ куб $[-R, R]^{3} \subset \mathbb{R}^{3}$ и разобьем его границу $\partial K$ на квадратики со стороной 2 и вершинами в множестве $(2 \mathbb{Z})^{3} \cap \partial K$. Далее, каждый такой квадратик разобьем на два треугольника так, чтобы для каждого треугольника $T$ этого разбиения существовала точка $x \in \mathbb{Z}^{3}$ со свойством $T^{(0)} \subset 2 E-x$, где $T^{(0)}$ обозначает множество вершин треугольника $T$. Используя включение (3), мы видим, что

$$
T^{(0)} \subset(2 \mathbb{Z})^{3} \backslash A_{i}
$$

где $i$ выбирается из условия $x \in A_{i}$. Таким образом, мы определили некоторую триангуляцию гранищы $\partial K$ куба $K$.

Обозначим через $\Delta$ двумерный симплекс с вершинами $v_{1}, v_{2}, v_{3}$. Построим кусочно-линейное отображение $f: \partial K \rightarrow \Delta$, определив его в вершинах триангуляции формулой $f(v)=v_{i}$, где $v \in A_{i}$. Из кусочной линейности отображения $f$ и соотношения (4) следует, что $f$ отображает границу $\partial K$ куба $K$ в границу $\partial \Delta$ симплекса $\Delta$. Рассмотрим плоскость $s=\left\{(x, y, z) \in \mathbb{R}^{3} \mid x=0\right\}$ и ограничение $f \mid \partial K \cap s$ отображения $f$ на "диаметр" $\partial K \cap s$ "сферы" $\partial K$. Поскольку “диаметр" $\partial K \cap s$ стягиваем в $\partial K$, отображение $f \mid \partial K \cap s: \partial K \cap s \rightarrow \partial \Delta$ гомотопически тривиально. Докажем, что это не так.

Повторяя аргументацию леммы 4 , можно доказать, что $f(x) \neq f(-x)$ для любой точки $a \in \partial K \cap s$.

Посредством любого гомеоморфизма отождествим $\partial \Delta$ с единичной окружностью $S^{1}=\{z \in \mathscr{C}:|z|=1\}$ на комплексной плоскости. Поскольку $|f(x)|=|f(-x)|=1$ 
и $f(x) \neq f(-x)$, то $f(x)-t f(-x) \neq 0$ для любых $x \in \partial K \cap s$ и $t \in[0,1]$. Тогда гомотопия $h:(\partial K \cap s) \times[0,1] \rightarrow \partial \Delta=S^{1}$, определенная формулой

$$
h(x, t)=\frac{f(x)-t f(-x)}{|f(x)-t f(-x)|},
$$

непрерьвна и соединяет отображение $f \mid \partial K \cap s=h_{0}$ с отображением

$$
h_{1}(x)=\frac{f(x)-f(-x)}{|f(x)-f(-x)|},
$$

которое, как легко убедиться, антиподально, т.е. $h_{1}(-x)=-h_{1}(x)$ для любого $x \in$ $\partial K \cap s$. Согласно [4, гл. IV,$\S 7.10]$ антиподальные отображения (в том числе отображение $h_{1}$ ) не гомотопически тривиальны. Получаем противоречие с гомотопической тривиальностью отображения $f \mid \partial K \cap s$.

ДоКАЗАТЕЛЬСТво ТЕОРЕмы 2. Теорема очевидньм образом следует из лемм $4-6$.

Перейдем теперь к доказательству теоремы 4 . Для каждого $n \geqslant 0$ рассмотрим подмножество $T_{n} \subset \mathbb{R}^{n}$, определенное следующим (индуктивным) образом: $T_{0}=\varnothing \subset \mathbb{R}^{0}=$ $\{0\}$ и $T_{n}=\mathbb{R}^{n-1} \times\{0\} \cup T_{n-1} \times[0, \infty) \subset \mathbb{R}^{n-1} \times \mathbb{R}=\mathbb{R}^{n}$ для $n \geqslant 1$.

Лемма 7. Для каждого $n \in \mathbb{N}$ существует разбиение $A_{1} \cup \cdots \cup A_{n}$ пространства $\mathbb{R} \times \mathbb{R}^{n-1}$, удовлетворяюшее следующим двум условиям:

i) для любих $1 \leqslant i \leqslant n$ и $a \in \mathbb{R} \times T_{n-1}$ пересечение $A_{i} \cap\left(2 a-A_{i}\right)$ ограничено;

ii) для любы $1 \leqslant i \leqslant n u b \in \mathbb{R} \times \mathbb{R}^{n-1}$ проекиия пересечения $A_{i} \cap\left(2 b-A_{i}\right)$ на $\mathbb{R}^{n-1}$ ограничена.

ДоКАЗАТЕЛЬСТво. Для $n=1$ лемма очевидна. Предположим, что для некоторого $n$ мы построили разбиение $\mathbb{R} \times \mathbb{R}^{n-1}=A_{1}^{\prime} \cup \cdots \cup A_{n}^{\prime}$, удовлетворяющее условиям леммы 7. Искомое разбиение $A_{0} \cup \cdots \cup A_{n}$ пространства $\mathbb{R} \times \mathbb{R}^{n}=\mathbb{R} \times \mathbb{R}^{n-1} \times \mathbb{R}$ определим следующим образом:

$$
\begin{gathered}
A_{0}=\bigcup_{t>0}[0, \infty) \times[-t, t]^{n-1} \times\{-t\} \cup \bigcup_{t, \tau \geqslant 0}\{-\tau\} \times[-t-\tau, t+\tau]^{n-1} \times\{-t\} \\
A_{i}=\left(A_{i}^{\prime} \times \mathbb{R}\right) \backslash A_{0}, \quad 1 \leqslant i \leqslant n .
\end{gathered}
$$

Несложно проверить, что это разбиение удовлетворяет условиям леммы 7.

Назовем подмножество $A$ группы $\mathbb{Z}^{n} T$-образным, если $f(A) \subset \mathbb{R} \times T_{n-1}$ для некоторого инъективного аффинного отображения $f: \mathbb{Z}^{n} \rightarrow \mathbb{R} \times \mathbb{R}^{n-1}$. Отметим, что подмножество группы $\mathbb{Z}^{2} T$-образно тогда и только тогда, когда оно содержится в некоторой прямой в плоскости $\mathbb{R}^{2} \supset \mathbb{Z}^{2}$; подмножество $A \subset \mathbb{Z}^{3} T$-образно, если $A \subset s \cup \pi_{+}$для некоторой плоскости $s \subset \mathbb{R}^{3} \supset \mathbb{Z}^{3}$ и замкнутой полуплоскости $\pi_{+}$в $\mathbb{R}^{3}$, пересекающейся c $s$ по граничной прямой $\partial \pi_{+}$.

Из леммы 7 вытекает следующая важная лемма.

Лемма 8. Т-образные подмножсества не существенны в $\mathbb{Z}^{n}$.

Следующая лемма дает полное описание структуры существенных подмножеств группы $\mathbb{Z}^{2}$. 
Лемма 9. Подмножество $A \subset \mathbb{Z}^{2}$ существенно тогда и лишь тогда, когда оно содержит три аффинно независимые точки.

ДокАЗАтЕЛЬСтво. Пусть $A$ - существенное подмножество групшы $\mathbb{Z}^{2}$. Согласно лемме 8 множество $A$ не $T$-образно. Тогда $A$ не содержится в прямой и, следовательно, содержит три аффинно независимые точки.

Покажем теперь, что любое подмножество $A \subset \mathbb{Z}^{2}$, содержащее три аффинно независимые точки $a_{0}, a_{1}, a_{2}$, существенно в $\mathbb{Z}^{2}$. Зафиксируем любое разбиение $\mathbb{Z}^{2}=A_{1} \cup A_{2}$ группы $\mathbb{Z}^{2}$ на два подмножества. Пусть $f: \mathbb{Z}^{2} \rightarrow \mathbb{Z}^{2}-$ (единственное) аффинное отображение, удовлетворяющее условию $f\left(e_{i}\right)=a_{i}, i=1,2,3$, где $e_{0}=(0,0), e_{1}=(1,0)$ и $e_{2}=(0,1)$. Поскольку точки $a_{0}, a_{1}, a_{2}$ аффинно независимы, отображение $f$ инъективно. Из доказательства леммы 4 следует существование таких $i \in\{1,2\}$ и $j \in\{0,1,2\}$, что множество $f^{-1}\left(A_{i}\right) \cap\left(2 e_{j}-f^{-1}\left(A_{i}\right)\right)$ бесконечно. Ввиду инъективности отображения $f$ множество $A_{i} \cap\left(2 a_{j}-A_{i}\right) \supset f\left(f^{-1}\left(A_{i}\right) \cap\left(2 e_{j}-f^{-1}\left(A_{i}\right)\right)\right)$ тоже бесконечно, т.е. $A-$ существенное подмножество $\mathbb{Z}^{2}$.

Теперь мы займемся изучением существенных подмножеств групшы $\mathbb{Z}^{3}$, которую мы отождествляем с подмножеством в $\mathbb{R}^{3}$.

ЛЕМма 10. Октаэдры и только они являются существенными подмножествами $\mathbb{Z}^{3}$ мошиности $\leqslant 6$.

ДокАЗАтЕльство. Предположим, что $A=\left\{a \pm e_{1}, a \pm e_{2}, a \pm e_{3}\right\} \subset \mathbb{Z}^{3}$ - октаэдр (здесь $a \in \mathbb{R}^{3}$ и $e_{1}, e_{2}, e_{3}$ - линейно независимые векторы в $\mathbb{R}^{3}$ ). Мы покажем, что $A$ - существенное подмножество в $\mathbb{Z}^{3}$. Отметим, что существенное подмножество $E=\{0,1\}^{3} \backslash\{(0,1,1),(1,0,0)\}$ из доказательства леммы 6 является октаэдром $\left\{a^{\prime} \pm e_{i}^{\prime}: i=1,2,3\right\}$, где

$$
a^{\prime}=\left(\frac{1}{2}, \frac{1}{2}, \frac{1}{2}\right), \quad e_{1}^{\prime}=\left(-\frac{1}{2},-\frac{1}{2},-\frac{1}{2}\right), \quad e_{2}^{\prime}=\left(-\frac{1}{2}, \frac{1}{2},-\frac{1}{2}\right), \quad e_{3}^{\prime}=\left(-\frac{1}{2},-\frac{1}{2}, \frac{1}{2}\right) .
$$

Определим аффинное отображение $f: \mathbb{R}^{3} \rightarrow \mathbb{R}^{3}$, положив $f\left(a^{\prime}\right)=a$ и $f\left(a^{\prime}+e_{i}^{\prime}\right)=a+e_{i}$ для $i=1,2,3$. Легко видеть, что отображение $f$ инъективно. Отметим, что

$$
\begin{aligned}
f(0,0,0) & =f\left(a^{\prime}+e_{1}^{\prime}\right)=a+e_{1} \in \mathbb{Z}^{3}, \\
f(0,1,0) & =f\left(a^{\prime}+e_{2}^{\prime}\right)=a+e_{2} \in \mathbb{Z}^{3}, \\
f(0,0,1) & =f\left(a^{\prime}+e_{3}^{\prime}\right)=a+e_{3} \in \mathbb{Z}^{3}, \\
f(1,1,1) & =f\left(2 a^{\prime}+\left(a^{\prime}+e_{2}^{\prime}\right)+\left(a^{\prime}+e_{3}^{\prime}\right)-3\left(a^{\prime}+e_{1}^{\prime}\right)\right) \\
& =2 f\left(a^{\prime}\right)+f\left(a^{\prime}+e_{2}^{\prime}\right)+f\left(a^{\prime}+e_{3}^{\prime}\right)-3 f\left(a^{\prime}+e_{1}^{\prime}\right) \in \mathbb{Z}^{3} .
\end{aligned}
$$

Отсюда следует, что $f\left(\mathbb{Z}^{3}\right) \subset \mathbb{Z}^{3}$. Отметим также, что

$f\left(a^{\prime}-e_{i}^{\prime}\right)=f\left(2 a^{\prime}-\left(a^{\prime}+e_{i}^{\prime}\right)\right)=2 f\left(a^{\prime}\right)-f\left(a^{\prime}+e_{i}^{\prime}\right)=2 a-\left(a+e_{i}\right)=a-e_{i}, \quad i=1,2,3$.

Следовательно, $f(E)=A$.

Покажем, что множество $A$ существенно в $\mathbb{Z}^{3}$. Пусть $\mathscr{U}$ - покрытие групшы $\mathbb{Z}^{3}$ мощности $|\mathcal{U}|<4=\nu\left(\mathbb{Z}^{3}\right)$. Тогда $f^{-1}(\mathscr{U})=\left\{f^{-1}(U) \mid U \in \mathscr{U}\right\}-$ покрытие группы $\mathbb{Z}^{3}$ мощности $\left|f^{-1}(\mathscr{U})\right|<4$. Из доказательства леммы 7 следует, что множество $E$ существенно в $\mathbb{Z}^{3}$. Стало быть, существует точка $e \in E$ и множество $U \in \mathscr{U}$ такие, что пересечение $f^{-1}(U) \cap\left(2 e-f^{-1}(U)\right)$ бесконечно. Ввиду инъективности отображения $f$ 
пересечение $U \cap(2 f(e)-U) \supset f\left(f^{-1}(U) \cap(2 e-U)\right)$ тоже бесконечно. Следовательно, множество $A=f(E) \ni f(e)$ существенно в $\mathbb{Z}^{3}$.

Покажем теперь, что любое существенное подмножество группы $\mathbb{Z}^{3}$, содержащее не более шести точек, является октаэдром. Обозначим через $\operatorname{conv}(A)$ вьпуклую оболочку множества $A$ в $\mathbb{R}^{3}$. Зафиксируем любую грань многогранника $\operatorname{conv}(A)$ и обозначим через $s$ плоскость, в которой эта грань лежит. Поскольку $|A| \leqslant 6$, за пределами плоскости лежит не более трех точек множества $A$. Поскольку плоскость $s$ опорная для выпуклого множества $\operatorname{conv}(A)$, множество $A \backslash s$ содержится в одном из полупространств относительно плоскости $s$. Обозначим это полупространство через $v_{+} \cdot$ Если существует плоскость $\pi$, содержащая множество $A \backslash s$ и пересекающая плоскость $s$, то в силу леммы 8 множество $A$ несущественно, поскольку оно содержится в $T$-образном подмножестве $s \cup\left(v_{+} \cap \pi\right)$. Отсюда мы делаем вывод, что $|A|=6,|A \backslash s|=3$ и (единственная) плоскость, содержащая множество $A \backslash s$, параллельна плоскости $s$.

Таким образом, $\operatorname{conv}(A)$ - выпукльй многогранник, каждая грань которого содержит ровно три точки множества $A$; остальные три точки множества $A$ образуют параллельную грань. Несложно показать, что октаэдры - единственные выпуклые многогранники с шестью вершинами, обладающие этим свойством.

ЛЕмма 11. Существует минимальное существенное конечное подмножество группье $\mathbb{Z}^{3}$ мошности $>\chi\left(\mathbb{Z}^{3}\right)$.

ДокАЗАтЕльство. Рассмотрим отображения $f_{i}: \mathbb{Z}^{2} \rightarrow \mathbb{Z}^{3}, i=1,2,3$, определенные формулами

$$
f_{1}(x, y)=(-1, x, y), \quad f_{2}(x, y)=(x,-1, y), \quad f_{3}(x, y)=(x, y,-1)
$$

Повторяя доказательство леммы 6 , можно показать, что объединение

$$
A=\bigcup_{1 \leqslant i \leqslant 3} f_{i}\left(\{0,1\}^{2}\right)
$$

трех квадратиков существенно в $\mathbb{Z}^{3}$. Множество $A$ содержит некоторое минимальное существенное множество $B$. Легко видеть, что множество $A$ не содержит октаэдров. Следовательно, $B$ - не октаэдр и по лемме $10|B|>6$.

ДокАЗАТЕЛЬСТво тЕОРЕмы 4. Теорема очевидным образом следует из лемм 9-11.

Наиболее интересным и интригуюшим открытым вопросом в данной тематике является вычисление конкретных значений $\chi\left(\mathbb{Z}^{n}\right)$ для $n>3$. Отметим, что естественная (ввиду теоремы 2 ) оптимистическая гипотеза $\chi\left(\mathbb{Z}^{n}\right)=n(n+1) / 2$ неверна в силу следующего предложения.

ПРЕДЛОЖЕНИЕ. Имеет место неравенство $\chi\left(\mathbb{Z}^{4}\right)>10$.

ДокАЗАТЕльство. В силу леммы 8 достаточно доказать, что любое десятиэлементное подмножество $A$ группы $\mathbb{Z}^{4} T$-образно. Можно рассуждать следующим образом. Рассмотрим выпуклую оболочку $\operatorname{conv}(A)$ множества $A$ в $\mathbb{R}^{4} \supset \mathbb{Z}^{4}$. Несложно найти опорную к $\operatorname{conv}(A)$ трехмерную гиперплоскость $s \subset \mathbb{R}^{4}$, содержащую не менее четырех точек множества $A$. Тогда $|A \backslash s| \leqslant 6$. Можно предполагать, что дополнение $A \backslash s$ непусто. (Если $A \backslash s=\varnothing$, то все множество $A$ содержится в гиперплоскости $s$ и, как легко убедиться, является $T$-образным множеством в $\mathbb{Z}^{4}$.) Рассмотрим выпуклую оболочку $\operatorname{conv}(A \backslash s)$ множества $A \backslash s$ в $\mathbb{R}^{4}$. Можно предполагать, что существует такая 
опорная к $\operatorname{conv}(A \backslash s)$ гиперплоскость $p$, что $s \cap p \neq \varnothing$ и $|A \backslash(s \cup p)| \leqslant 2$. (Предположение отсутствия такой гиперплоскости $p$ влечет $A \backslash s \subset s^{\prime}$ для некоторого трехмерного аффиннного подпространства, не пересекающего $s$. Меняя, если нужно, $s$ и $s^{\prime}$ ролями, мы можем добиться, чтобы $|A \backslash s| \leqslant 5$. В этом случае можно провести любую опорную к $\operatorname{conv}(A \backslash s)$ гиперплоскость $p$ такую, что $p \cap s \neq \varnothing$ и $|(A \backslash s) \backslash p| \leqslant 2$.) Зафиксируем, наконец, любую гиперплоскость $q \subset \mathbb{R}^{4}$, содержащую множество $A \backslash(s \cup p)$ и пересекающую гиперплоскости $s$ и $p$. Обозначим через $s_{-}, s_{+}, p_{-}, p_{+}, q_{-}, q_{+}$открытые полупространства в $\mathbb{R}^{4}$ относительно гиперплоскостей $s, p, q$ соответственно, причем $v_{-} \cap A=\varnothing$, $p_{-} \cap(A \backslash s)=\varnothing, q_{-} \cap(A \backslash(s \cup p))=\varnothing$. Легко видеть, что

$$
\mathbb{Z}^{4} \cap\left(s \cup\left(p \cap v_{+}\right) \cup\left(q \cap v_{+} \cap p_{+}\right)\right)
$$

является $T$-образным множеством в $\mathbb{Z}^{4}$, содержащим множество $A$.

Вышеприведенные рассуждения а также определение $T$-образных множеств наталкивают на следующий вопрос.

Вопрос 1. Справедливо ли неравенство $\chi\left(\mathbb{Z}^{n}\right) \geqslant n(n-1)$ для $n \in \mathbb{N} ?$ Более конкретно, является ли $T$-образньм любоеподмножество $A \subset \mathbb{Z}^{n}$ мощности $|A|<n(n-1)$ ?

Можно показать, что любое минимальное существенное подмножество четырехмерного куба $\{0,1\}^{4} \subset \mathbb{Z}^{4}$ содержит в точности четырнадцать точек (выброшены две диагональные точки). Следовательно, $11 \leqslant \chi\left(\mathbb{Z}^{4}\right) \leqslant 14$. Отметим, что в отличие от групп $\mathbb{Z}^{n}$, $1 \leqslant n \leqslant 3$, в $\mathbb{R}^{4}$ не существует правильного вьпуклого многогранника, множество вершин которого является минимальным сушественным подмножеством группы $\mathbb{Z}^{4}$ (поскольку правильные многогранники в $\mathbb{R}^{4}$ содержат $8,16,24,120$ либо 600 вершин [5]).

Вот еще некоторые другие вопросы, касающиеся проблематики данной статьи.

Вопрос 2. Справедливо ли строгое неравенство $\chi\left(\mathbb{Z}^{n}\right)<\chi\left(\mathbb{Z}^{n+1}\right)$ для каждого $n$ ?

Вопрос 3 . Верна ли теорема 3 для групп $G$ произвольной мощности?

Вопрос 4 . Пусть $G$-группа с конечньп числом элементов порядка $2, H$-подгруппа группы $G$ и $A$ - существенное подмножество в $G$. Верно ли, что образ $A$ при факторотображении $G \rightarrow G / H$ является существенным множеством в $G / H$ ?

Вопрос 5. Существует ли существенное подмножество группы $\mathbb{Z}^{3}$, не содержащее конечных существенных подмножеств?

Вопрос 6 . Каждое ли существенное подмножество групшы $\mathbb{Z}^{3}$ содержит минимальное существенное подмножество?

Вопрос 7 . Существуют ли в $\mathbb{Z}^{3}$ минимальные существенные подмножества сколь угодно большой мощности?

\section{СПИСОК ЦИТИРОВАННОЙ ЛИТЕРАТУРЫ}

[1] Банах Т.О., Протасов И.В. Асимметричные разбиения абелевых групп // Матем. заметки (в печати).

[2] Протасов И.В. Асимметрично разложимые абелевы группы // Матем. заметки. 1996. T. 59. № 3. С. 468-471.

[3] Фукс Л. Бесконечные абелевы группы. Т. І. М.: Мир, 1970.

[4] Спеньер Э. Алгебраическая топология. М.: Мир, 1971.

[5] Люстерник Л. А. Выпуклые фигуры и многогранники. М.: Гостехиздат, 1956. 